Revista Complutense de Ciencias Veterinarias

ISSN-e 1988-2688

\title{
Modelos económicos de simulación en el cebo de terneros
}

\author{
Daza Andrada, Argimiro ${ }^{1}$
}

\begin{abstract}
Resumen. Se realiza un análisis de simulación en el cebo de terneros, machos y hembras, introduciendo en el modelo como variables independientes influyentes el precio de compra del kilogramo del ternero destetado, el precio de venta del kilogramo canal del ternero cebado, el precio del pienso y el índice de conversión del pienso y como variables dependientes la ganancia, el margen bruto y la renta disponible por animal. Las hembras logran mayores ganancias, márgenes brutos y rentas disponibles que los machos. Las variables independientes que más contribuyen a la varianza de las variables dependientes citadas fueron el precio de compra del kilogramo de ternero destetado y el precio del kilogramo canal de venta del ternero cebado mientras que el índice de conversión del pienso es la variable de menor contribución a la varianza.
\end{abstract}

Palabras clave: costes; ganancia; ingresos; margen bruto.

\section{[en] Economical models of simulation in the calves fattening}

\begin{abstract}
In the present work a simulation analysis for the calves fattening (males anf females) was achieved. In the simulation model were introduced as independent variables the purchase price of kilogram of weaned calf, sale price of carcass kilo of fattened calf, feed price and the conversion rate of feed, whereas the profit, gros margin and disposable income were considered as dependent variables. Females obtained higher profits, gross margins and disposable income than the males. The independent variables that had the highest contribution to the variance of dependent variables were the purchase and sale price of half, whereas the variable that had the lowest contribution to the variance was the conversion rate of feed.
\end{abstract}

Key words: costs; profit; income; gros margin.

\section{Introducción}

En los resultados económicos del cebo de terneros influyen un conjunto de factores técnicos: tipo genético, sexo, peso de los terneros al inicio del cebo, peso al sacrificio, índice de conversión del pienso, rendimiento a la canal y condiciones ambientales (Daza, 2014). Los principales factores de influencia inherentes a la esfera económica son el precio de compra y de venta del ternero y el precio del pienso, factores que varían con el tiempo según coyuntura económica.

Algunos trabajos previos, realizados en España, han estudiado modelos de simulación dirigidos hacia la gestión técnico-económica del ganado vacuno de carne en extensivo (Bernués et al.2015), pero no relacionados con el cebo intensivo de terneros basado en la estabulación y la alimentación con paja y concentrados. Sin embargo, un breve trabajo preliminar, que consideró el citado segmento productivo, donde se estudió el efecto sobre la ganancia de las variables económicas precio de compra del ternero destetado y el de venta del ternero cebado fue realizado por Daza (2007). Dada la importancia que reviste el tema para los cebaderos españoles, en general, y para los ubicados en el ecosistema de la Dehesa en particular, y como quiera que se dispone de escasa información al respecto, el objetivo de este experimento es establecer modelos económicos de simulación para el cebo de terneros, machos y hembras, utilizando las tres variables económicas precio de compra del ternero, precio de venta del mismo y precio del pienso y la variable técnica índice de conversión del pienso.

\footnotetext{
Departamento de Producción Agraria (Producción Animal).

Escuela Técnica Superior de Ingeniería Agronómica, Alimentaria y de Biosistemas.

Universidad Politecnica de Madrid.

Ciudad Universitaria 28040 Madrid, España.
} 


\section{Material y Métodos}

El estudio observa los datos técnicos y económicos del último año (2017), extraídos de proyectos profesionales, cooperativas ganaderas y de la Lonja de Precios de Extremadura, correspondientes a terneros cruzados Limusín $\mathrm{x}$ (Charolés $\mathrm{x}$ Retinta) cebados en el término municipal de Cáceres y localidades próximas .

\subsection{Hipótesis de cálculo}

\subsubsection{Machos}

En la hipótesis de cálculo para los terneros machos (Tabla 1) se considera que el peso medio inicial de los terneros es de $220 \mathrm{~kg}$, el peso final de venta $550 \mathrm{~kg}$, la ganancia media diaria de $1,4 \mathrm{~kg}$ y el rendimiento a la canal fría el $56 \%$ con lo que el tiempo de ocupación, si el tiempo de limpieza y desinfección es de 4-5 días, es de 240 días (Tabla 1). Se estima que el coste de inversión de la plaza (alojamiento e instalaciones), según proyectos de ingeniería agronómica, es de $800 €$, el valor de desecho de la plaza $80 €$ y la vida útil de la misma 40 años. El coste anual en conservación y reparaciones se asume que es el $1,5 \%$ del coste de inversión, que una unidad técnica agraria (UTA) supone para la empresa (sueldo y seguridad social) $18.000 € /$ año y que la UTA tiene capacidad para llevar 1000 cabezas. Los costes higiosanitarios, según información veterinaria de la zona, y los costes varios por plaza y ciclo se estiman en 22 y $20 €$ respectivamente. En el coste de alimentación se considera que el consumo de paja por plaza es del $15 \%$ del consumo de pienso (Bacha 2002) y que el coste de la paja es de $0,07 € / \mathrm{kg}$. Para el cálculo de los intereses de alojamientos e instalaciones y del ternero para cebo el tipo de interés anual se asume que es del 1\%. El cálculo de los intereses del capital circulante (conservación y reparaciones, mano de obra, higiosanitarios, varios y alimentación) observa el pago a mes vencido (por ello se divide el coste correspondiente por dos) y un interés anual también del $1 \%$.Debe quedar claro que los intereses de alojamientos e instalaciones, del ternero y del capital circulante son considerados como costes de oportunidad y no como costes financieros. Los costes de compra y venta del ternero consideran un IVA del 10,5\% y los costes de conservación y reparaciones, alimentación, higiosanitarios y varios incluyen el IVA correspondiente. En los ingresos no se considera la subvención por ternero de la UE.

Tabla 1. Hipótesis de cálculo y estimación de la ecuación de la ganancia para terneros machos

\begin{tabular}{|c|c|c|}
\hline COSTES & HIPÓTESIS DE CÁLCULO & $\begin{array}{c}\text { CÁLCULO DEL COSTE } \\
\text { POR PLAZA, POR TENERO } \\
\text { E INGRESOS POR TENERO }\end{array}$ \\
\hline $\begin{array}{l}\text { Amortización de alojamientos e } \\
\text { instalaciones }\left(\mathrm{A}_{\mathrm{a}}\right)\end{array}$ & $\begin{array}{l}\text { Coste plaza ternero }=800 € \\
\text { Valor desecho plaza }=80 € \\
\text { Vida útil plaza }=40 \text { años }\end{array}$ & $\begin{array}{l}A_{a}=(800-80) /(40 \times 1,52)= \\
11,84 € .\end{array}$ \\
\hline Conservación y reparaciones (CR) & $\begin{array}{l}1,5 \% \text { de la inversión } \\
\text { Interés }=1 \% \text { anual } \\
\text { Conservación y reparaciones } \\
\text { se paga a mes vencido }\end{array}$ & $\begin{array}{l}\mathrm{CR}=(800 \times 0,015) / 1,52=7,89 € . \\
\text { Interés }=[7,89 /(2 \times 1,52)] \times 0,01= \\
0,026 . € . \\
\text { Total } \mathrm{CR}=7,89+0,026=7,92 € .\end{array}$ \\
\hline Mano de obra (MO) & $\begin{array}{l}18.000 € / \text { UTA y año } \\
1 \text { UTA asiste a } 1000 \text { terneros } \\
\text { Interés } 1 \% \\
\text { Mano de obra se paga a mes vencido }\end{array}$ & $\begin{array}{l}\mathrm{MO}=18.000 /(1000 \times 1,52)=11,84 € . . \\
\text { Interés }=[11,84 /(2 \times 1,52)] \times 0,01=0,039 \\
€ . \\
\text { Total } \mathrm{MO}=11,84+0,039=11,88 € .\end{array}$ \\
\hline Higiosanitarios $(\mathrm{H})$ & $\begin{array}{l}22 € \text { por plaza y ciclo coste de } \\
\text { desparasitaciones, vacunaciones } \\
\text { y desinfección de alojamientos e } \\
\text { instalaciones } \\
\text { Interés }=1 \% \\
\text { Higiosanitarios se pagan a mes } \\
\text { vencido }\end{array}$ & $\begin{array}{l}\mathrm{H}=22 € \\
\text { Interés }=[22 /(2 \times 1,52)] \times 0,01=0,072 € . \\
\text { Total } \mathrm{H}=22+0,072=22,07 €\end{array}$ \\
\hline
\end{tabular}




\begin{tabular}{|c|c|c|}
\hline COSTES & HIPÓTESIS DE CÁLCULO & $\begin{array}{c}\text { CÁLCULO DEL COSTE } \\
\text { POR PLAZA, POR TENERO } \\
\text { E INGRESOS POR TENERO }\end{array}$ \\
\hline Varios (V) & $\begin{array}{l}20 € \text { por plaza y ciclo en agua, } \\
\text { energía, cama de paja, impuestos, } \\
\text { seguros, transporte, oficina etc } \\
\text { Interés }=1 \% \\
\text { Varios se pagan a mes vencido }\end{array}$ & $\begin{array}{l}\mathrm{V}=20 € \\
\text { Interés }=[20 /(2 \times 1,52)] \times 0,01=0,066 € \\
\text { Total } \mathrm{V}=20,06 €\end{array}$ \\
\hline Alimentación $(\mathrm{A})^{*}$ & $\begin{array}{l}\text { El consumo de paja es el } 15 \% \text { del } \\
\text { consumo de pienso. } \\
\text { Precio de la paja }=0,07 € / \mathrm{kg} \text {. } \\
\text { Interés }=1 \% \\
\text { La alimentación se paga a mes } \\
\text { vencido }\end{array}$ & $\begin{array}{l}\mathrm{A}=(550-220) \mathrm{kg} \times \mathrm{IC} \mathrm{kg} / \mathrm{kg} \times \mathrm{PP} € / \mathrm{kg}+ \\
(550-220) \times \mathrm{IC} \mathrm{kg} / \mathrm{kg} \times .0,15 \times 0,07 € / \mathrm{kg} . \\
\text { Interés }=[\mathrm{A} /(2 \times 1,52)] \times 0,01 \\
\text { Total } \mathrm{A}=\mathrm{A}+[\mathrm{A} /(2 \times 1,52)] \times 0,01=(331 \\
\times \mathrm{IC} \times \mathrm{PP}+3,48) €\end{array}$ \\
\hline Coste del ternero CT & $\begin{array}{l}\text { Peso inicial del ternero }=220 \mathrm{~kg} \\
\text { Interés }=1 \% \\
\mathrm{IVA}=10,5 \%\end{array}$ & $\begin{array}{l}\mathrm{CT}=220 \mathrm{~kg} \times \mathrm{PC} € / \mathrm{kg}+220 \mathrm{~kg} \text { x PC x } \\
0,105=243,1 \times \mathrm{PC} \\
\text { Interés }=[(243,1 \times \mathrm{PC}) / 1,52] \mathrm{x} 0,01=1,6 \times \mathrm{PC} \\
\text { Total CT }=(243,1 \times \mathrm{PC})+1,6 \times \mathrm{PC}= \\
244,7 \times \mathrm{PC}\end{array}$ \\
\hline $\begin{array}{l}\text { Interés de alojamientos e } \\
\text { instalaciones }\left(\mathrm{I}_{\mathrm{a}}\right)\end{array}$ & Interés $=1 \%$ & $I_{a}=[(800+80) /(2 \times 1,52)] x \cdot 0,01=2,89 €$ \\
\hline Total/plaza y ciclo & & $\begin{array}{l}\text { Total PLAZA }=76,668 €+331,085 x x \text { IC } \\
x \mathrm{PP}+3,48 \times \mathrm{IC}+244,7 \times \mathrm{PC}\end{array}$ \\
\hline Total ternero $(\mathrm{TT})$ & La mortalidad de terneros es del $0,5 \%$ & TT $(€)=$ Total PLAZA/0,995 \\
\hline INGRESOS/ternero $(\mathrm{G})$ & $\begin{array}{l}\text { El rendimiento a la canal fría es del } \\
56 \% . \mathrm{IVA}=10,5 \% \\
\text { No se considera la subvención de la } \\
\text { UE }\end{array}$ & $\begin{array}{l}\mathrm{G}=550 \mathrm{~kg} \times 0,56 \times \mathrm{PV} €+\mathrm{IVA}=308 \times \\
\mathrm{PV} €+\mathrm{IVA}=308 \times \mathrm{PV}+0,105 \times 308 \\
\mathrm{PV}=340,34 \times \mathrm{PV} .\end{array}$ \\
\hline
\end{tabular}

$\mathrm{IC}=$ índice de conversión del pienso en $\mathrm{kg} / \mathrm{kg}, \mathrm{PP}=$ precio del pienso de cebo en $€ / \mathrm{kg}, \mathrm{PC}=$ precio en $€$ del $\mathrm{kg}$ de ternero destetado con $220 \mathrm{~kg}$ de peso medio, $\mathrm{PV}=$ precio del $\mathrm{kg}$ canal en $€$ del ternero cebado vendido. Los costes incluyen el IVA correspondiente.1,52 $=\mathrm{n}^{\mathrm{o}}$ de ciclos por año $(\mathrm{NC})$, cifra a la que se llega mediante la expresión: $\mathrm{NC}=$ 365 días/[[(550-220)/1,4 kg/día] + 4,3 días de limpieza y desinfección $]=365 /(235,71+4,29$ días $)=(365$ días/año)/ (240 días/ciclo) =1,52 ciclos/año, siendo, $220 \mathrm{~kg}, 550 \mathrm{~kg}$ y 1,4 kg/día el peso medio inicial de los terneros, el peso final de venta y la ganancia media diaria media durante el periodo de cebo respectivamente. * A $=$ (peso final terneropeso inicial ternero). indice de conversión del pienso. ( precio del $\mathrm{kg}$ de pienso en $€+0,15$. precio del $\mathrm{kg}$ de paja en $€)$. Los intereses de los componentes del capital circulante: conservación y reparaciones, alimentación, mano de obra, higiosanitarios y varios se han calculado dividiendo el coste correspondiente entre el producto 2. 1,52 y el resultado obtenido multiplicado por el interés anual asumido.

\subsubsection{Hembras}

En la hipótesis de cálculo para las hembras (Tabla 2) se considera que el peso medio inicial de las terneras es de $220 \mathrm{~kg}$, el peso final de venta $450 \mathrm{~kg}$, la ganancia media diaria de $1,15 \mathrm{~kg}$ y el rendimiento a la canal fría el $55 \%$ con lo que el tiempo de ocupación, si el tiempo de limpieza y desinfección es de 4 días, es de 204 días (Tabla 2) Se asume, así mismo, que el coste de la plaza en el cebadero de hembras es de $700 €$ (el cebo de hembras necesita menos superficie disponible por animal que el de machos 5 versus $6 \mathrm{~m}^{2}$ por animal) el valor de desecho $70 €$ y la vida útil 40 años. Para los costes restantes se consideran datos similares a los considerados para los machos. Se estima que el índice de transformación del pienso de los machos entre 220 y $550 \mathrm{~kg}$ es similar al de las hembras entre 220 y $450 \mathrm{~kg}$ (las hembras comienzan a depositar grasa más tempranamente que los machos). (Daza 1999, 2014) 
Tabla 2. Hipótesis de cálculo y estimación de la ecuación de la ganancia para las hembras

\begin{tabular}{|c|c|c|}
\hline COSTES & HIPÓTESIS DE CÁLCULO & $\begin{array}{c}\text { CÁLCULO DEL COSTE } \\
\text { POR PLAZA, POR TENERO } \\
\text { E INGRESOS POR TERNERO }\end{array}$ \\
\hline $\begin{array}{l}\text { Amortización de alojamientos e } \\
\text { instalaciones }\left(\mathrm{A}_{\mathrm{a}}\right)\end{array}$ & $\begin{array}{l}\text { Coste plaza ternera }=700 € \\
\text { Valor desecho plaza }=70 € \\
\text { Vida útil plaza }=40 \text { años }\end{array}$ & $A_{a}=(700-70) /(40 \times 1,79)=\mathbf{8 , 8} €$ \\
\hline Conservación y reparaciones (CR) & $\begin{array}{l}1,5 \% \text { de la inversión } \\
\text { Interés }=1 \% \text { anual } \\
\text { Conservación y reparaciones } \\
\text { se paga a mes vencido }\end{array}$ & $\begin{array}{l}\mathrm{CR}=(700 \times 0,015) / 1,79=5,9 € \\
\text { Interés }=[5,9 /(2 \times 1,79)] \times 0,01= \\
0,016 . € \\
\text { Total CR }=5,9+0,016=5,92 €\end{array}$ \\
\hline Mano de obra (MO) & $\begin{array}{l}18.000 € / \text { UTA y año } \\
1 \text { UTA asiste a } 1000 \text { terneras } \\
\text { Interés } 1 \% \\
\text { Mano de obra se paga a mes vencido }\end{array}$ & $\begin{array}{l}\mathrm{MO}=18.000 /(1000 \times 1,79)=10,05 € \\
\text { Interés }=[10,05 /(2 \times 1,79)] \times 0,01=0,028 € \\
\text { Total } \mathrm{MO}=10,05+0,028=10,08 €\end{array}$ \\
\hline Higiosanitarios $(\mathrm{H})$ & $\begin{array}{l}22 € \text { por plaza y ciclo coste de } \\
\text { desparasitaciones, vacunaciones } \\
\text { y desinfección de alojamientos e } \\
\text { instalaciones } \\
\text { Interés }=1 \% \\
\text { Higiosanitarios se pagan a mes vencido }\end{array}$ & $\begin{array}{l}\mathrm{H}=22 € \\
\text { Interés }=[22 /(2 \times 1,79)] \times .0,01=0,06 € \\
\text { Total } \mathrm{H}=22+0,06=22,06 €\end{array}$ \\
\hline Varios $(\mathrm{V})$ & $\begin{array}{l}20 € \text { por plaza y ciclo en agua, energía, } \\
\text { cama de paja, impuestos, seguros, } \\
\text { transporte, oficina etc } \\
\text { Interés }=1 \% \\
\text { Varios se pagan a mes vencido }\end{array}$ & $\begin{array}{l}\mathrm{V}=20 € \\
\text { Interés }=[20 /(2 \times 1,79)] \times 0,01=0,055 € \\
\text { Total } \mathrm{V}=20,05 €\end{array}$ \\
\hline Alimentación $(\mathrm{A}) *$ & $\begin{array}{l}\text { El consumo de paja es el } 15 \% \text { del } \\
\text { consumo de pienso. } \\
\text { Precio de la paja }=0,07 € / \mathrm{kg} \text {. } \\
\text { Interés }=1 \% \\
\text { La alimentación se paga a mes vencido }\end{array}$ & $\begin{array}{l}\mathrm{A}=(450-220) \mathrm{kg} \times \mathrm{IC} \mathrm{kg} / \mathrm{kg} \times \mathrm{PP} € / \mathrm{kg} \\
+(450-220) \times \mathrm{IC} \mathrm{kg} \times 0,15 \times 0,07 € / \mathrm{kg} \\
\text { Interés }=[\mathrm{A} /(2 \times 1,79)] \times 0,01 \\
\text { Total } \mathrm{A}=\mathrm{A}+[\mathrm{A} /(2 \times 1,79)] \times 0,01= \\
230,64 \mathrm{IC} \times \mathrm{PP}+2,422 \times \mathrm{IC}\end{array}$ \\
\hline $\begin{array}{l}\text { Coste del ternero } \\
\text { (CT) }\end{array}$ & $\begin{array}{l}\text { Peso inicial de la ternera }=220 \mathrm{~kg} \\
\text { Interés }=1 \% \\
\mathrm{IVA}=10,5 \%\end{array}$ & $\begin{array}{l}\mathrm{CT}=220 \mathrm{~kg} \times \mathrm{PC} € / \mathrm{kg}+220 \mathrm{~kg} \times \mathrm{PC} \times \\
0,105=243,1 \times \mathrm{PC} \\
\text { Interés }=[(243,1 \times \mathrm{PC}) / 1,79] \times 0,01= \\
1,358 \mathrm{PC} \\
\text { Total CT }=(243,1 \times \mathrm{PC})+1,358 \times \mathrm{PC} \\
=244,46 \times \mathrm{PC}\end{array}$ \\
\hline $\begin{array}{l}\text { Interés de alojamientos e } \\
\text { instalaciones }\left(\mathrm{I}_{\mathrm{a}}\right)\end{array}$ & Interés $=1 \%$ & $I_{a}=[(700+70) /(2 \times 1,79] \times \cdot 0,01=2,15 €$ \\
\hline Total/plaza y ciclo & & $\begin{array}{l}\text { Total PLAZA }=69,06 €+230,64 \times \text { IC } \\
\times \mathrm{PP}+2,422 \times \mathrm{IC}+244,46 \times \mathrm{PC}\end{array}$ \\
\hline Total ternera $(\mathrm{TT})$ & La mortalidad de terneras es del $0,5 \%$ & TT $(€)=$ Total PLAZA/0,995 \\
\hline $\begin{array}{l}\text { INGRESOS/ternera } \\
(\mathrm{G})\end{array}$ & $\begin{array}{l}\text { El rendimiento a la canal fría es del } \\
56 \% . \mathrm{IVA}=10,5 \% \\
\text { No se considera la subvención de la } \\
\text { UE }\end{array}$ & $\begin{array}{l}G=450 \mathrm{~kg} \times 0,55 \times \mathrm{PV} € \mathrm{IVA}=247,5 \\
\times \mathrm{PV} € \times 0,105=25,99 \times \mathrm{PV} . \\
\mathrm{G} \text { total }=273,49 \times \mathrm{PV} .\end{array}$ \\
\hline
\end{tabular}

$\mathrm{IC}=$ índice de conversión del pienso en $\mathrm{kg} / \mathrm{kg}, \mathrm{PP}=$ precio del pienso de cebo en $€ / \mathrm{kg}, \mathrm{PC}=$ precio en $€$ del $\mathrm{kg}$ de la ternera destetada, $\mathrm{PV}=$ precio del $\mathrm{kg}$ canal en $€$ de la ternera cebada. Los costes incluyen el IVA correspondiente.

$1,79=\mathrm{n}^{\circ}$ de ciclos por año (NC), cifra a la que se llega mediante la expresión: $\mathrm{NC}=365$ días/[[(450-220)/1,15 kg/día] + 4 días de limpieza y desinfección $]=365 /(200+4$ días $)=(365$ días/año $) /(204$ días $/$ ciclo $)=1,79$ ciclos $/$ año, siendo , $220 \mathrm{~kg}, 450 \mathrm{~kg}$ y $1,15 \mathrm{~kg} /$ día el peso medio inicial de las terneras, el peso final de venta y la ganancia media diaria media durante el periodo de cebo respectivamente.

* $\mathrm{A}=$ (peso final ternero-peso inicial ternero). indice de conversión del pienso. ( precio del $\mathrm{kg}$ de pienso en $€+0,15$. precio del kg de paja en $€$ ). Los intereses de los componentes del capital circulante: conservación y reparaciones, alimentación, mano de obra, higiosanitarios y varios se han calculado dividiendo el coste correspondiente entre el producto 2. 1,52 y el resultado obtenido multiplicado por el interés anual asumido. 


\subsection{Cálculos y análisis estadísticos}

El cálculo de costes e ingresos se ha llevado a cabo según el método propuesto por Alonso y Serrano $(1991,2004)$ y la ganancia se calculó como la diferencia de ingresos y costes. Considerando valores de las variables $\mathrm{PC}=$ $3,1,3,2,3,3,3,4$ y $3,5 € / \mathrm{kg}$ para los machos y $2,2,2,3,2,4,2,5$ y $2,6 € / \mathrm{kg}$ para las hembras, $\mathrm{PV}=3,7,3,8,3,9,4,0$ y $4,1 € / \mathrm{kg}$ canal, $\mathrm{PP}=0,20,0,21,0,22,0,23$ y $0,24 € / \mathrm{kg}$, e IC $=4,6,4,7,4,8,4,9,5,0 \mathrm{~kg} / \mathrm{kg}$ para machos $\mathrm{y}$ hembras se elaboró un archivo EXCEL de 625 filas $(5 \times 5 \times 5 \times 5)$.. Mediante la Hoja de Cálculo EXCEL se calcularon las variables dependientes GA (ganancia), MB (margen bruto) y RD (renta disponible), y mediante el paquete de regresión del SG Centurión se obtuvieron las ecuaciones de regresión múltiple entre las precitadas variables dependientes y las variables independientes, PC, PV, PP e IC.

Adicionalmente se realizó un análisis de componentes de varianza, mediante el paquete estadístico SG Centurión, con el fin de estimar la cantidad con la que contribuyen a la varianza de las variables dependientes las diferentes variables independientes consideradas.

\section{Resultados y Discusión}

Según los datos aportados en la Tabla 1 la ecuación de la ganancia respondió a la ecuación:

$$
\begin{gathered}
\mathrm{GA}(€)=340,34 \times \mathrm{PV}-(76,7+331,1 \times \mathrm{IC} \\
\mathrm{x} \mathrm{PP} .+3,48 \times \mathrm{IC}+244,7 \times \mathrm{PC}) / 0,995
\end{gathered}
$$

$\mathrm{Si}$ consideramos el margen bruto (MB) como la diferencia entre ingresos y costes pero sin incluir en los costes la amortización de alojamientos e instalaciones y los intereses, la ecuación del $\mathrm{MB}$ respondería a la expresión:

$\mathrm{MB}(€)=340,34 \times \mathrm{PV}-(61,73+243,1 \times$ $\mathrm{PC}+330 \times \mathrm{IC} \times \mathrm{PP}+3,465 \times \mathrm{IC}) / 0,995$.

Así mismo, si consideramos la renta disponible (RD) como la diferencia entre el $\mathrm{MB}$ y el coste de la mano de obra (MO) (caso en el que el propietario constituye la mano de obra directa de la explotacisón) la ecuación correspondiente a la $\mathrm{RD}$ respondería a la expresión:

$\mathrm{RD}=\mathrm{MB}-\mathrm{MO}=340,34 \times \mathrm{PV}-(49,89+$ $243,1 \times \mathrm{PC}+330 \times \mathrm{IC} \times \mathrm{PP}+3,465 \times \mathrm{IC}) / 0,995$

En la Tabla 3 reflejamos las ecuaciones de regresión múltiple entre las variables dependientes e independientes indicadas para los terneros machos

Tabla 3. Ecuaciones de regresión múltiple entre las variables dependientes GA, MB y RD y las dependientes PC, PV, PP e IC para los machos

\begin{tabular}{|l|c|c|c|}
\hline Ecuación de regresión & $\mathrm{R}^{2}$ & $\mathrm{RSD}$ & $\mathrm{P}<$ \\
\hline $\begin{array}{l}\mathrm{GA}=(274,31 \pm 1,38)+(340,34 \pm 0,19) \times \mathrm{PV}-(245,93 \pm 0,19) \times \mathrm{PC}-(1597, \\
25 \pm 1,89) \times \mathrm{PP}-(76,70 \pm 0,19) \times \mathrm{IC}\end{array}$ & 0,99 & 0,66 & 0,0001 \\
\hline $\begin{array}{l}\mathrm{MB}=(288,19 \pm 1,38)+(340,34 \pm 0,19) \times \mathrm{PV}-(244,32 \pm 0,19) \times \mathrm{PC}-(1591, \\
96 \pm 1,88) \times \mathrm{PP}-(76,45 \pm 0,19) \times \mathrm{IC}\end{array}$ & 0,99 & 0,66 & 0,0001 \\
\hline $\begin{array}{l}\mathrm{RD}=(300,09 \pm 1,38)+(340,34 \pm 0,19) \mathrm{PV}-(244,32 \pm 0,19) \mathrm{PC}-(1591,96 \\
\pm 1,88) \mathrm{PP}-(76,45 \pm 0,19) \mathrm{IC}\end{array}$ & 0,99 & 0,66 & 0,0001 \\
\hline
\end{tabular}

$\mathrm{n}=625, \mathrm{R} 2=$ coeficiente de determinación, $\mathrm{RSD}=$ desviación residual estándar, $\mathrm{P}=$ probabilidad. Valor des estadístico de Mallows $=5,0 \mathrm{GA}=$ ganancia, $\mathrm{PC}=$ Precio del $\mathrm{kg}$ de compra del ternero destetado, $\mathrm{PV}=$ Precio del $\mathrm{kg}$ de venta del ternero cebado, $\mathrm{PP}=$ precio del $\mathrm{kg}$ de pienso, $\mathrm{IC}=$ índice de conversión del pienso expresado en $\mathrm{kg} / \mathrm{kg}$.

Como cabía esperar, conjuntamente, las variables PV, PC, PP e IC explicaron el 99\% de la variabilidad de la GA, MB y RD siendo las regresiones muy significativas.
En la Tabla 4 aparecen los resultados del análisis de componentes de varianza correspondiente a los machos 
Tabla 4. Análisis de componentes de varianza para los machos

\begin{tabular}{|l|l|c|}
\hline \multicolumn{1}{|c|}{ Variable dependiente } & \multicolumn{1}{|c|}{ Variable independiente } & $\%$ \\
\hline \multirow{4}{*}{ GANANCIA } & Precio kg compra ternero destetado & 20,93 \\
\cline { 2 - 3 } & Precio kg venta ternero cebado & 62,11 \\
\cline { 2 - 3 } & Precio del pienso & 13,65 \\
\cline { 2 - 3 } & Índice de conversión del pienso & 3,31 \\
\hline \multirow{4}{*}{$\begin{array}{l}\text { MARGEN BRUTO y RENTA } \\
\text { DISPONIBLE }\end{array}$} & Precio kg compra ternero destetado & 20,60 \\
\cline { 2 - 3 } & Precio kg venta ternero cebado & 62,46 \\
\cline { 2 - 3 } & Precio del pienso & 13,63 \\
\cline { 2 - 3 } & Índice de conversión del pienso & 3,31 \\
\hline
\end{tabular}

Como puede observarse en la Tabla 3 las variables independientes PV y PC son las que más contribuyen a la varianza de las variables dependientes GA, MB y RD, mientras que el IC es la que menos contribuye.

Según los datos aportados en la Tabla 2, la ecuación de la ganancia para las hembras respondió a la ecuación:

GA $(€)=273,49 \times \mathrm{PV}-(69,06+230,64 \times$ IC x PP. +2,422 x IC+244. 46 x PC)/0,995.
Y de la misma manera que para los machos las ecuaciones obtenidas correspondientes al margen bruto y la renta disponible fueron:

$\mathrm{MB}(€)=273,49 \times \mathrm{PV}-(57,95+243,1 \times$ $\mathrm{PC}+230 \times \mathrm{IC} \times \mathrm{PP}+2,415 \times \mathrm{IC}) / 0,995$.

$\mathrm{RD}=\mathrm{MB}-\mathrm{MO}=273,49 \times \mathrm{PV}-(47,90+$ $243,1 \times \mathrm{PC}+230 \times \mathrm{IC} \times \mathrm{PP}+2,415 \times \mathrm{IC}) / 0,995$

En la Tabla 5 reflejamos las ecuaciones de regresión múltiple entre las variables dependientes e independientes indicadas para las hembras

Tabla 5. Ecuaciones de regresión múltiple entre las variables dependientes GA, MB y RD y las dependientes PC, PV, PP e IC para las hembras

\begin{tabular}{|c|c|c|c|}
\hline Ecuación de regresión & $\mathrm{R}^{2}$ & RSD & $\mathrm{P}<$ \\
\hline $\begin{array}{l}\mathrm{GA}=(175,37 \pm 0,92)+(273,49 \pm 0,13) \times \mathrm{PV}-(245,69 \pm 0,13) \times \mathrm{PC}- \\
1112,64 \pm 1,32) \times \mathrm{PP}-(53,43 \pm 0,13) \times \mathrm{IC}\end{array}$ & 0,99 & 0,47 & 0,0001 \\
\hline $\begin{array}{l}\mathrm{MB}=(185,85 \pm 0,92)+(273,49 \pm 0,13) \times \mathrm{PV}-(244,32 \pm 0,13) \times \mathrm{PC}-( \\
1109,52 \pm 1,31) \times \mathrm{PP}-(53,28 \pm 0,13) \times \mathrm{IC}\end{array}$ & 0,99 & 0,46 & 0,0001 \\
\hline $\begin{array}{l}\mathrm{RD}=(195,90 \pm 0,92)+(273,49 \pm 0,13) \times \mathrm{PV}-(244,32 \pm 0,13) \times \mathrm{PC}-( \\
1109,52 \pm 1,31) \times \mathrm{PP}-(53,28 \pm 0,13) \times \mathrm{IC}\end{array}$ & 0,99 & 0,46 & 0,0001 \\
\hline
\end{tabular}

$\mathrm{n}=625, \mathrm{R} 2=$ coeficiente de determinación, $\mathrm{RSD}=$ desviación residual estándar, $\mathrm{P}=$ probabilidad. Valor del estadístico de Mallows $=5,0 . \mathrm{GA}=$ ganancia, $\mathrm{PC}=$ Precio del $\mathrm{kg}$ de compra del ternero destetado, $\mathrm{PV}=$ Precio del $\mathrm{kg}$ de venta del ternero cebado, $\mathrm{PP}=$ precio del $\mathrm{kg}$ de pienso, $\mathrm{IC}=$ índice de conversión del pienso.

Como en el caso de los machos, conjuntamente, las variables PV, PC, PP e IC explicaron el $99 \%$ de la variabilidad de la GA, MB y $\mathrm{RD}$ siendo las regresiones muy significativas.
El análisis de componentes de varianza correspondiente a las hembras aparece en la Tabla 6 obteniéndose valores en la línea de los obtenidos para los machos. 
Tabla 6. Análisis de componentes de varianza

\begin{tabular}{|l|l|c|}
\hline \multicolumn{1}{|c|}{ Variable dependiente } & \multicolumn{1}{|c|}{ Variable independiente } & $\%$ \\
\hline \multirow{4}{*}{ GANANCIA } & Precio kg compra ternero destetado & 34,29 \\
\cline { 2 - 3 } & Precio kg venta ternero cebado & 54,62 \\
\cline { 2 - 3 } & Precio del pienso & 8,92 \\
\cline { 2 - 3 } & Índice de conversión del pienso & 2,16 \\
\hline \multirow{4}{*}{$\begin{array}{l}\text { MARGEN BRUTO y } \\
\text { RENTA DISPONIBLE }\end{array}$} & Precio kg compra ternero destetado & 33,98 \\
\cline { 2 - 3 } & Precio kg venta ternero cebado & 54,94 \\
\cline { 2 - 3 } & Precio del pienso & 8,92 \\
\cline { 2 - 3 } & Índice de conversión del pienso & 2,16 \\
\hline
\end{tabular}

Si comparamos la ganancia margen bruto y renta disponible entre machos y hembras, considerando el precio de compra del $\mathrm{kg}$ de peso vivo de los terneros al destete $(3,45 € / \mathrm{kg}$ para los machos y $2,60 € / \mathrm{kg}$ para las hembras), el precio de venta del $\mathrm{kg}$ canal $(3,85 € / \mathrm{kg}$ tanto para machos como para hembras), el precio actual (año 2018) del pienso de cebo más frecuente en los cebaderos $(0,22 € / \mathrm{kg})$ y un índice de conversión del pienso de $5,0 \mathrm{~kg} / \mathrm{kg}$, tanto en machos como en hembras, característico de terneros cruzados, sustituyendo tales valores en las ecuaciones de regresión de las Tablas 3 y 5 obtenemos que la GA, MB y RD ascienden para los machos a $1,26,23,11$ y $35,01 €$ respectivamente, mientras que para las hembras se alcanzan las cifras de 77,58, 93,06 y 103,11 $€$ respectivamente resultados que demuestran la baja rentabilidad actual del cebo de terneros machos y la apreciable rentabilidad del cebo de hembras. Daza (2007) también obtuvo una mayor rentabilidad con el cebo de hembras que con el de machos en el escenario productivo del ecosistema de la Dehesa española don- de los precios del pienso y de la paja suelen ser más elevados que en las zonas cerealistas de España.

\section{Conclusiones}

La rentabilidad del cebo de hembras es superior a la del cebo de machos debido al menor coste de adquisición de las mismas para el cebo. Resultaría interesante para los cebaderos que estimaran ecuaciones como las calculadas en este trabajo, útiles para recabar información sobre la rentabilidad por ternero y para realizar análisis de sensibilidad haciendo variar los valores de las variables independientes introducidas en los modelos. De otra parte cabe señalar que los modelos de simulación pueden considerar variables independientes adicionales a las observadas en este estudio (peso al sacrificio, peso de inicio del cebo, etc), lo que, sin duda, complicaría y alargaría el proceso de cálculo, aunque incrementaría el interés de este tipo de trabajos.

\section{Referencias bibliográficas}

Alonso, R. y Serrano, A. (1991). Los costes en los procesos de producción agraria. Ed Mundi Prensa, 147 pp.

Alonso, R. y Serrano, A. (2004). Economía de la empresa agroalimentaria. Ed Mundi Prensa, 383 pp.

Bacha, F. (2002). Nutrición, patología digestiva y salud intestinal de rumiantes en cebo, aspectos prácticos. XVIII Curso de Especialización FEDNA. Avances en Nutrición y Alimentación Animal, pp142-160. Barcelona, 4-5 de noviembre de 2002 Ed FEDNA.

Bernués, A. 2015. Modelos de simulación para la gestión técnico-económica del ganado vacuno de carne en extensivo. Ponencia XX Congreso Internacional ANEMBE, Burgos 7-8 de mayo de 2015.

Daza, A. (2007). Efecto del sexo sobre los resultados económicos del cebo de terneros. ITEA, Vol Extra, 28, Tomo I: 336-338.

Daza, A. (1999). Producción extensiva de vacuno de carne en la Dehesa. Madrid, Ed Luzán, 100pp.

Daza, A. (2014). Vacuno de carne con criterios económicos. Madrid Ed Agrícola Española SA y MAPAMA, $320 \mathrm{pp}$. 